\title{
Probing the Reaction Mechanism for Highly Reactive Nanothermite Formulations
}

\author{
Rohit J. Jacob ${ }^{1}$, Wen-An Chiou ${ }^{2}$ and Michael R. Zachariah ${ }^{3}$ \\ 1. Dept. of Chemical and Biomolecular Eng., University of Maryland, College Park, MD 20742 \\ 2. NISP Lab, NanoCenter, University of Maryland, College Park, MD 20742-2831, USA \\ 3. Depts. of Chemical and Biomol. Eng. and Chemistry, Univ. of Maryland, College Park, MD 20742
}

Formulations of aluminum nano particles for energy applications have been intensely investigated over the past few decades for their attractive energy release rate. They significantly affect propellant burn rates, enhance detonation properties, and are also used in thermite formulations ("Super-Thermites") [1]; however, the reactive properties remain poorly understood. The mechanism behind these energetic systems is currently under debate. On one hand, Al nano particles possess a protective alumina shell in their native state leading to the belief of the inter-diffusion mechanism (oxidizer and fuel through shell) during reaction [2]. On the other hand, a more violent mechanism where the particle shell disintegrates upon the melting of the aluminum core has also been proposed [3]. This study used electron microscopy to probe the underlying mechanism through a forensic analysis of the products of these reactions.

Al nano particles (ALEX) were procured from Argonide Corp., the copper oxide nano powder was obtained from Sigma Aldrich. Those particles were suspended in hexane and ultrasonicated for 20 minutes to ensure intimate mixing which were then pipetted onto a fine platinum wire $(76 \mu \mathrm{m})$. The wire was heated to $1400 \mathrm{~K}$ at $\sim 2 \times 10^{5} \mathrm{~K} / \mathrm{sec}$ to ignite the particles. The ignition products were subsequently captured directly on SEM stubs which were kept at a separation of $1 \mathrm{~mm}$ from the wire. Similarly, ignition products were collected on $\mathrm{C}$-coated $\mathrm{Cu}$ grids for TEM study. Selected particles of products were cut using FIB technique for cross-section SEM investigation.

SEM image of $\mathrm{Al} / \mathrm{CuO}$ mixture before combustion shows very fine spherical particles ranging between 5 to $10 \mathrm{~nm}$ for $\mathrm{CuO}$ and 50 to $100 \mathrm{~nm}$ for $\mathrm{Al}$, and interspersed with large aggregates (50 to $300 \mathrm{~nm}$ ) (Fig. 1). Particle size of products (after reaction) drastically increased to $5 \mu \mathrm{m}$ or even $\sim 10 \mu \mathrm{m}$ though a fair amount of $1 \mu \mathrm{m}$ particles was also visible (Fig. 2). TEM images of the A1/CuO particles after combustion depicted very similar results. High magnification TEM images revealed another population of particles in sub-micron range (Fig. 3). The particle surface of products revealed a completely different aggregate morphology. Nearly all large particles of products, regardless of size, are coated with a thin layer of particles (Figs. 2, 4, 5 and 6). A fair amount of fine particles in the range of $<50 \mathrm{~nm}$ was observed, consistent with the average size of a (Smoluchowski) population that can grow from the gas phase using simple aerosol growth calculations. Those small particles likely resulted from a gas phase reaction whereas the larger particles resulted from a reaction in the condensed phase, termed as Reactive Sintering [4]. Particle size distribution (using Image J) showed that the larger particles constituted about $90 \%$ of the products. This result indicates that the predominant reaction follows a condensed phase route where the reactants sintered in the early stage of the reaction and the fuel and oxidizer diffuse toward each other [4]. EDS analysis of small particles in TEM and of a cross-sectional SEM sample (Fig. 6) depicts that $\mathrm{O}$ content $(\sim 10 \%)$ was far below expected $(40 \%)$ if fully reacted, i.e., the reaction was only $25 \%$ complete. This brings to question the advantages of using nanoscale reactants as the current evidence suggests that they are sintering into much larger structures before the reaction completes. 
[1] M.M. Mench et. al, Combust. Sci. and Tech. 135 (1998), p. 269-292.

[2] G. Jian et. al, J. Phys. Chem. C 116 (2012), p. 26881-26887.

[3] V. I. Levitas et. al, Appl. Phys. Lett. 89 (2006), p. 071909.

[4] K. T. Sullivan et. al, Combust. Flame 159 (2012), p. 2-15;

[5] This research was supported by Army Research Office and the Defense Threat Reduction Agency, and also partially supported by NSF-MRSEC (DMR 05-20471) and UMD.

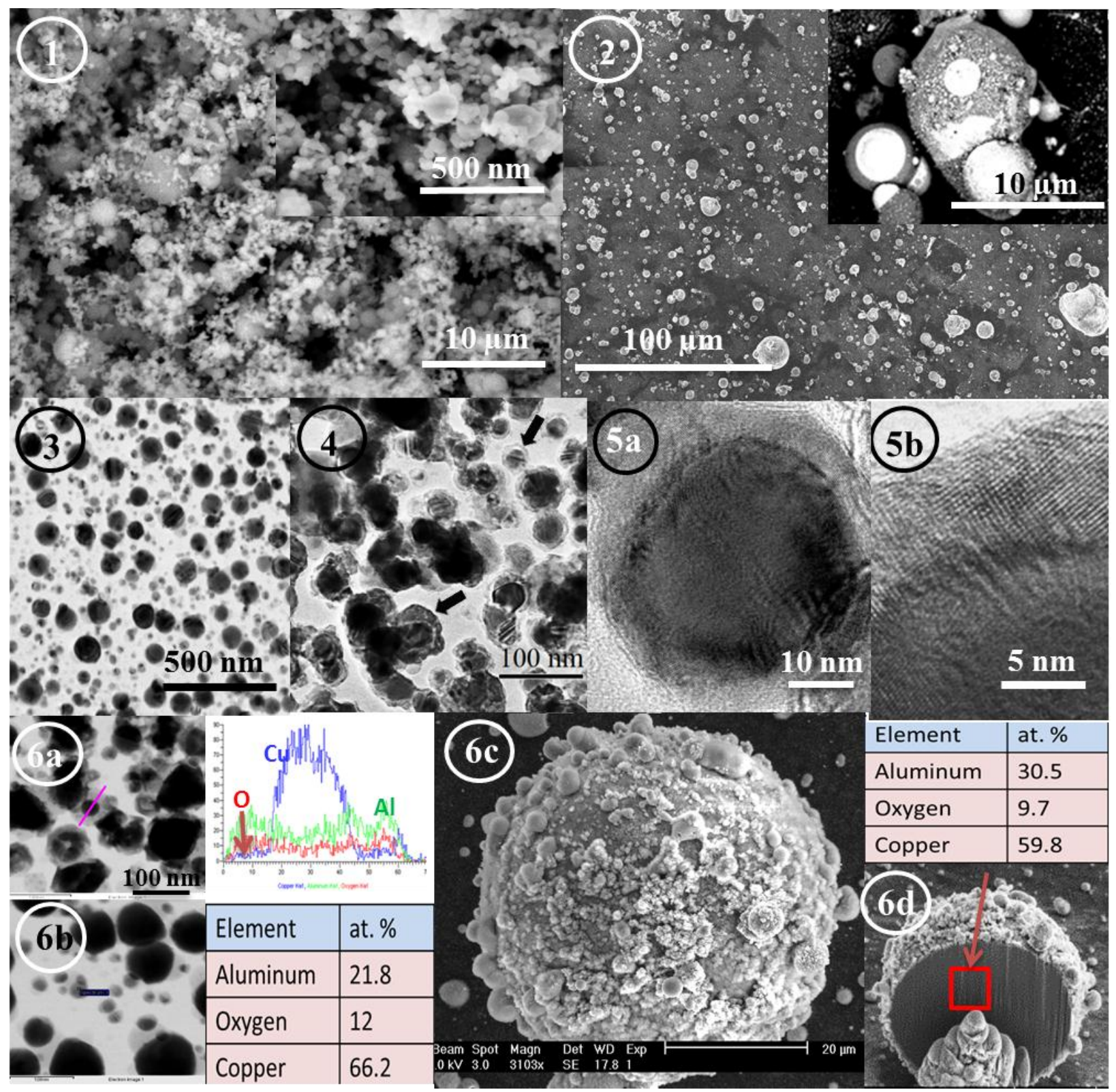

Fig. 1. SEM images of nano reactants show fine spherical particles of $\mathrm{Al}$ (small) and $\mathrm{CuO}$ (large). Fig. 2. SEM images of nano $\mathrm{Al} / \mathrm{CuO}$ products depict increased particle size. Note the scale bar. Fig. 3. TEM micrograph of products reveals fine particles population in addition to large particles. Fig. 4. TEM image shows coatings (arrows) on the particle surface and aggregation of particles. Fig. 5. HRTEM image of a particle $(\sim 50 \mathrm{~nm})$ reveals shelled structure (a) and surface coating (b). Fig. 6. TEM EDS line profile (a) and spot (b) analyses of a core shelled structure, and EDS area analysis of FIB cross-sectioned sample (c and d) indicate incomplete combustion reaction. 